\title{
Cooperative Multi-hop Relaying via Network Formation Games in Cognitive Radio Networks
}

\author{
Wei Li*, Xiuzhen Cheng*, Tao Jing ${ }^{\dagger}$, Xiaoshuang Xing ${ }^{\dagger}$ \\ ${ }^{*}$ Computer Science, The George Washington University, Washington DC, USA \\ ${ }^{\dagger}$ School of Electronics and Information Engineering, Beijing Jiaotong University, Beijing, China \\ Email: \{weili, cheng\}@gwu.edu, \{tjing, 10120170\}@bjtu.edu.cn
}

\begin{abstract}
The cooperation between the primary and the secondary users has attracted a lot of attention in cognitive radio networks. However, most existing research mainly focuses on the single-hop relay selection for a primary transmitter-receiver pair, which might not be able to fully explore the benefit brought by cooperative transmissions. In this paper, we study the problem of multi-hop relay selection by applying the network formation game. In order to mitigate interference and reduce delay, we propose a cooperation framework FTCO by considering the spectrum sharing in both the time and the frequency domain. Then we formulate the multi-hop relay selection problem as a network formation game, in which the multi-hop relay path is computed via performing the primary player's strategies in the form of link operations. We also devise a distributed dynamic algorithm PRADA to obtain a global-path stable network. Finally, we conduct extensive numerical experiments and our results indicate that cooperative multi-hop relaying can significantly benefit both the primary and the secondary network, and that the network graph resulted from our PRADA algorithm can achieve the global-path stability.
\end{abstract}

Index Terms-Cognitive radio networks; cooperative multi-hop relaying; network formation game; global-path stable network.

\section{INTRODUCTION}

In cognitive radio networks, the mutual beneficial relationship between the primary users (PUs) and the secondary users (SUs) has been exploited to establish cooperative transmissions for performance enhancement [1]-[6]. With cooperation, a PU can increase its primary transmission rate through the relay service provided by a SU while the SU obtains channel access opportunities as a reward, which can simultaneously improve the performance of both the primary and the secondary network, achieving a "win-win" situation.

In this paper, we study the problem of multi-hop relay selection via a network formation game based on the combined cooperation in both the time and the frequency domain in a cooperative cognitive radio network (CCRN). Our consideration is motivated by the following observations.

- First, existing research mainly focuses on the single-hop relay selection, in which a primary transmitter (PT) employs relays to form one or multiple two-hop paths toward the primary receiver (PR) [1]-[6]. Nevertheless, in some network scenarios such as cognitive sensor networks, the SUs have to reduce their transmission ranges in order to conserve power and maintain a long-term lifetime. Under such a situation, only one relay on a path may not satisfy the requirement of the PU; thus finding a number of necessary relays for a primary transmitter-receiver pair becomes a key problem.
- Second, many existing cooperation frameworks are based on the idea of time slot division [1]-[5], in which the channel access time is divided into time slots allocated to both the primary traffic and the secondary traffic. However, such a model brings a waiting delay to the PUs and the relays, since a relay has to alternately forward the primary traffic and send its secondary traffic on the same channel. Thus, designing an effective cooperation model plays an important role in the performance enhancement of cooperative networks.

- Third but not the last, most game theory-based approaches in current research [2]-[6] provide mechanisms to determine the set of relays without pointing out the relay path, which can not be applied to the multi-hop relay selection.

To overcome the challenges mentioned above, we propose a novel solution in this paper. First, we devise a cooperation framework FTCO, in which the primary traffic and the secondary traffic are separated in the frequency domain and the relays share the leased sub-channel in the time domain, to mitigate interference and reduce delay for both the primary network and the secondary network. Second, we model the multi-hop relay selection problem as a network formation game, in which each primary player selfishly performs its strategies to form a multi-hop path towards its receiver to maximize its payoff. This process is summarized by a distributed stochastically dynamic algorithm PRADA, which yields a stable network graph. Finally, we validate the effectiveness of the PRADA algorithm and the stability of the resultant network graph via both theoretical analysis and numerical experiments. Our multifold contributions are summarized as follows:

- Different from the traditional cooperation frameworks, FTCO considers the cooperative transmissions in both the time and the frequency domain, allowing relays to forward the primary traffic and to send their secondary traffic at the same time without interfering with the PUs.

- To our best knowledge, we are the first to apply the network formation game to model the multi-hop relay selection in a CCRN. In the proposed network formation game, the player's payoff depends on not only the cooperation partners, but also the interconnections between them, indicating the impact of the communication path on the network performance.

- We propose a distributed dynamic network formation algorithm PRADA and mathematically prove that the network graph output by PRADA can achieve the global- 
path stability, which is equivalent to the Nash equilibrium in the proposed network formation game.

- Extensive numerical study confirms that a PU can benefit from a multi-hop relay path and a SU can gain an impressive throughput in our cooperative framework. Furthermore, the network formation process of PRADA can converge to output a global-path stable network graph.

The rest of this paper is organized as follows. In Section II, we briefly summarize the related literature. Our system model and network formation game are introduced in Sections III and IV, respectively. The distributed dynamic formation algorithm PRADA together with the theoretical analysis are detailed in Section V. After presenting our simulation results in Section VI, we conclude this paper in Section VII.

\section{RELATED WORK}

In recent years, researchers have shown a great interest in developing cooperation frameworks for the primary and secondary users by using game theory-based approaches such as Stackelberg game [2], [3], auction [4], coalition game [5], [7], bargaining [6], and so on.

In [2], the cooperation is modeled as a Stackelberg game, in which a primary user leases the spectrum to secondary users in exchange in the form of the distributed space-time coding. The PU attempts to maximize its performance in terms of either data rate or probability of outage, while the SUs compete among themselves for transmission within the leased time-slot following a distributed power control mechanism. A similar model is considered by Zhang et. al. [3], in which a primary user utilizes a set of secondary users as relays to form multiple two-hop paths for its throughput improvement, while a secondary user can access a fraction of the channel time for its own data transmissions. In [4], Yang et. al. design TASC, an auction scheme for cooperative communications where wireless nodes can trade relay services to motivate the participation of relay nodes and source nodes in cooperative communications while preventing any agent from rigging its bid or ask to manipulate the market at the same time. Li et. al. [5] exploit a transferable utility coalition game to model the cooperation in cooperative cognitive radio networks. Its results indicate that there is an operating point that maximizes the sum utility over all operators while providing each player a share to guarantee the stability of the grand coalition. $\mathrm{Xu}$ et. al. [6] propose a flexible channel cooperation based on Nash bargaining, which allows secondary users to freely use channels for transmitting primary data to the same base station along with their own data toward the secondary access point for the performance maximization.

As one of the most powerful tools in game theory, the network formation game, which represents the interconnections between players in a graph format, has been used to construct and analyze network graphs [8]-[13]. In [8], an agent-based computational network formation model is proposed for the Internet at the Autonomous System (AS) level based on realistic provider and peering strategies with ASs acting in a myopic and decentralized manner to optimize a cost-related fitness function. Nama et al. [9] use a non-cooperative bilateral connection game framework to study network formation of ad-hoc networks among selfish energy-constrained wireless devices that are interested in being connected with other devices. In their framework, devices choose their individual strategies to remain connected by minimizing only their direct transmission power costs. In addition, a number of previous research [10][12] presents different analytical network formation models to simulate the interactions between nodes during the network formation process, and to investigate the stability, connectivity, convergence, and efficiency of the network graphs.

To our best knowledge, the most related literature is [13], in which Saad et. al. formulate a network formation game among the relay stations in a $802.16 \mathrm{j}$ network, with an objective of setting up paths from relay stations to the base station to output a connected directed uplink tree network rooted at the base station. In this paper, we consider a two-tier network that supports the coexistence of the PUs and SUs, in which each primary transmitter has its own distinct receiver. We intend to establish cooperative communications between the PUs and SUs via multi-hop paths from the primary transmitters to their corresponding receivers for performance improvement. As a matter of fact, when both the PUs and the SUs act as players, a network formation game can be viewed as a multi-leader multi-follower Stackelberg game in which the PUs and SUs are leaders and followers, respectively. This brings unique challenges in devising the cooperation model, constructing the network formation game, and designing the formation algorithm, as the diversity of the primary transmission pairs also needs to be considered. Thus the network formation game proposed in [13] cannot be applied in this study.

\section{SySTEM MODEL}

\section{A. Network Model}

We consider a multi-hop cooperative cognitive radio network consisting of $N$ SUs, denoted by $\mathrm{SU}_{1}, \mathrm{SU}_{2}, \cdots, \mathrm{SU}_{N}$. Coexisting with this $\mathrm{CRN}$ is an OFDMA-based primary wireless network that supports $M$ distinct primary transmitter-receiver pairs, denoted by $\left\{\mathrm{PT}_{1}, \mathrm{PR}_{1}\right\},\left\{\mathrm{PT}_{2}, \mathrm{PR}_{2}\right\}, \cdots,\left\{\mathrm{PT}_{M}, \mathrm{PR}_{M}\right\}$. Let $\mathcal{M}$ and $\mathcal{N}$ be the sets of $\mathrm{PU}$ transceiver pairs and SUs, respectively. Then we have $\left\{\mathrm{PT}_{i}, \mathrm{PR}_{i}\right\} \in \mathcal{M}, 1 \leq i \leq M, M=|\mathcal{M}|$, and $\mathrm{SU}_{j} \in \mathcal{N}, 1 \leq j \leq N, N=|\mathcal{N}|$. The cooperation mechanism between the primary and secondary network will be detailed in the next subsection. The primary spectrum band contains multiple orthogonal sub-carriers and each primary transmitter is allocated with a subset of the sub-carriers, forming a sub-channel, for its communications. For simplicity, we assume that each sub-channel has $C$ sub-carriers, where $C$ is the same for all sub-channels; and each sub-carrier is assigned to at most one sub-channel, ensuring that multiple PTs can transmit simultaneously without inter-channel interference. Since our objective is to select relays for the primary communication pairs, the allocation of the sub-carriers to the PUs is out of the scope of this paper. For the same reason, we do not consider secondary traffic, i.e., the delivery of the data belonging to the cognitive radio network. We further assume that each SU has two radios, with one for relaying the primary traffic and one for simultaneously transmitting its own secondary traffic. 


\section{B. Cooperation Framework}

We adopt a simple cooperation framework in which PTs employ SUs to relay their data and provide channel access opportunities to SUs as rewards. With such a cooperative scheme, PTs can effectively transmit their data to a larger area and SUs can obtain more channel access opportunities, improving the performance of both the primary network and the secondary network. Our cooperation framework involves both the frequency domain and the temporal domain, which is termed Frequency and Time Combined cOoperation (FTCO), and is illustrated in Fig 1.

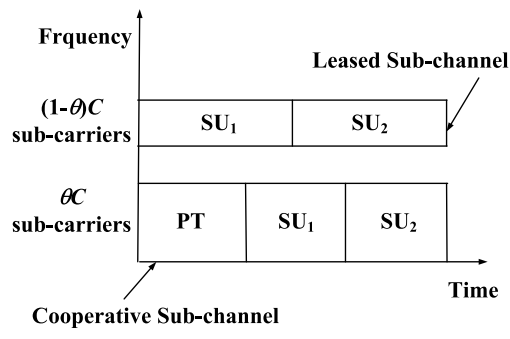

Fig. 1. The illustration of the FTCO framework

In FTCO, the $C$ sub-carriers of a sub-channel are divided into two parts: i) the set of $\theta C$ sub-carriers ${ }^{1}$ forming the cooperative sub-channel, which is used for the cooperative transmissions to support the primary traffic; and ii) the set of $(1-\theta) C$ sub-carriers called the leased sub-channel, which is allocated to the SUs as the reward according to time-based proportional fairness. Cooperative sub-channels are used by the PTs and SUs to transmit/relay the primary traffic only. For each relay, the times spent for receiving and forwarding the primary data are determined by the effective bit rates of the links on the path. To share the leased sub-channel, a unit access time is divided into multiple fractional portions each of which is assigned to a relay according to proportional fairness. The parameter " $\theta$ " indicates the tradeoff between a PT's bandwidth requirement and its cooperation degree. That is, the larger the value of $\theta$, the more bandwidth the PT requires and the smaller reward the relays receive. Such a cooperation framework has two nice features: on one hand, the primary traffic and the secondary traffic are separated in the frequency domain without inter-channel interference; on the other hand, the relays share the leased sub-channel in the time domain without interfering with each other.

\section{NETwORK Formation GAME Formulation}

To model the relay selection process in a multi-hop cooperative CRN, a network formation game is adopted, which is detailed in the following subsections.

\section{A. Players \& Network Graph}

In our network formation game, the players consist of the transmitters and the receivers of the primary communication pairs, and the SUs, which are denoted as primary players and secondary players, respectively (In this paper, primary

\footnotetext{
${ }^{1}$ if $\theta C$ is not an integer, choose the integer that is closest to $\theta C$.
}

transmitter/receiver and primary player are interchangeable; secondary user and secondary player are interchangeable.). All players are connected according to some network relationship summarized by a directed graph $G(V, E)$, with $V$ being the set of players, i.e., $V=\mathcal{M} \cup \mathcal{N}$, and $E$ being the set of links connecting two interacting players in the game. At the beginning of the game, $E=\emptyset$ since no relay (no communication link) has been selected for the primary transmitters.

Definition 1: (Path) A path of the $i$-th primary transmitterreceiver pair $L_{i}$ is defined as a subset of $E$ consisting of a sequence of players, i.e., $L_{i}=\left\{\left(v_{k}, v_{k+1}\right) \in E \mid k=\right.$ $\left.1,2, \cdots, K-1, v_{1}=\mathrm{PT}_{i}, v_{K}=\mathrm{PR}_{i}\right\}$, where $\left(v_{k}, v_{k+1}\right)$ is a directed link from $v_{k}$ to $v_{k+1}$ and $K$ is the number of players on the path.

Correspondingly, the set of players on a path $L_{i}$ is denoted by $V_{L_{i}}=\left\{v_{k} \mid\left(v_{k}, v_{k+1}\right)\right.$ or $\left(v_{k-1}, v_{k}\right) \in L_{i}, k=$ $1,2, \cdots, K\}$. Note that in this paper, we compute only one path for each primary transmitter-receiver pair and all the paths are node-disjoint.

\section{B. Payoff Functions}

According to our cooperation framework, a player benefits from participating in cooperative transmissions by forming links with others and also costs its own resources to maintain the links. Since PUs and SUs have different concerns such as effective bit rate and channel access time during the cooperation, our next step is to define the payoff functions to capture the incentives for PUs and SUs to cooperate.

Due to the limited transmit power, the effective bit rate between a PT and its PR may not satisfy the direct communication requirements. In the worse case, a PT cannot send packets to its PR via direct transmissions. Thus, the PT is motivated to employ relays for performance enhancement [14]. Assume that the bandwidth of each sub-carrier is $W \mathrm{~Hz}$; thus the effective bit rate of a sub-carrier in a link $(u, v)$ can be calculated by Shannon's theorem, i.e., $r_{(u, v)}=W \log _{2}\left(1+\operatorname{SNR}_{(u, v)}\right)$, where $\operatorname{SNR}_{(u, v)}$ is the Signal-to-Noise Ratio (SNR) received by $v$ from $u$. Accordingly, the effective bit rate of a sub-channel is $R_{(u, v)}=C \cdot r_{(u, v)}$. For direct transmissions, the path of the $i$-th primary pair is $L_{i}=\left\{\left(\mathrm{PT}_{i}, \mathrm{PR}_{i}\right)\right\}$, and the corresponding effective bit rate is,

$$
R_{i}(G)=C W \log _{2}\left(1+\mathrm{SNR}_{\left(\mathrm{PT}_{i}, \mathrm{PR}_{i}\right)}\right) .
$$

When the primary pair $\left\{\mathrm{PT}_{i}, \mathrm{PR}_{i}\right\}$ exploits cooperative transmissions, the effective bit rate of a link $\left(v_{k}, v_{k+1}\right)$ is,

$$
R_{\left(v_{k}, v_{k+1}\right)}(G)=\theta_{i} C W \log _{2}\left(1+\operatorname{SNR}_{\left(v_{k}, v_{k+1}\right)}\right),
$$

and the corresponding effective bit rate of the path $L_{i}$ is,

$$
R_{L_{i}}(G)=\min _{\left(v_{k}, v_{k+1}\right) \in L_{i}}\left\{R_{\left(v_{k}, v_{k+1}\right)}\right\} .
$$

Compared with direct transmissions, multi-hop communications may incur an undesirable delay, which can be taken as the cost of the cooperation for the PUs [15]. According to [16], a packet service system can be modeled as a $M / G / 1$ queue in a data network. Thus, the delay of the direct transmission is computed by

$$
D_{i}(G)=\frac{\lambda_{i}}{2 \mu_{i}\left(\mu_{i}-\lambda_{i}\right)}+\frac{1}{\mu_{i}},
$$


where $\lambda_{i}$ is the packet arrival rate of $\mathrm{PT}_{i}$ and $\mu_{i}=\frac{R_{i}(G)}{L e n}$ is the service rate with Len being the length of a packet. On the other hand, the delay of the cooperative transmission is obtained by (5), where $\mu_{\left(v_{k}, v_{k+1}\right)}=\frac{R_{\left(v_{k}, v_{k+1}\right)}(G)}{\text { Len }}$.

$$
\begin{aligned}
D_{L_{i}}(G)= & \sum_{\left(v_{k}, v_{k+1}\right) \in L_{i}} D_{\left(v_{k}, v_{k+1}\right)}(G) \\
= & \sum_{\left(v_{k}, v_{k+1}\right) \in L_{i}}\left[\frac{\lambda_{i}}{2 \mu_{\left(v_{k}, v_{k+1}\right)}\left(\mu_{\left(v_{k}, v_{k+1}\right)}-\lambda_{i}\right)}\right. \\
& \left.+\frac{1}{\mu_{\left(v_{k}, v_{k+1}\right)}}\right]
\end{aligned}
$$

To guarantee that all packets from the previous-hop are sent to the next-hop on a path, we assume that $\mu_{i}>\lambda_{i}$ and $\mu_{\left(v_{k}, v_{k+1}\right)}>\lambda_{i}$.

Note that a primary pair intends to find a path with a higher bit rate and a lower delay. In this regard, the payoff of the primary pair $\left\{\mathrm{PT}_{i}, \mathrm{PR}_{i}\right\}$ is defined by (6), where $\frac{R_{L_{i}}(G)-R_{i}(G)}{R_{i}(G)}$ is the bit rate profit and $\frac{D_{L_{i}}(G)-D_{i}(G)}{D_{i}(G)}$ is the delay cost.

$U_{i}^{P}\left(L_{i}, G\right)= \begin{cases}0, & \theta_{i}=1 ; \\ \frac{R_{L_{i}}(G)-R_{i}(G)}{R_{i}(G)}-\frac{D_{L_{i}}(G)-D_{i}(G)}{D_{i}(G)}, & 0<\theta_{i}<1 .\end{cases}$

This payoff function indicates that if $\theta_{i}=1$, the primary pair prefers direct transmissions without cooperating with others; otherwise when $0<\theta_{i}<1$, the primary pair selects SUs as relays to accomplish the cooperative transmissions. Moreover, if $U_{i}^{P}\left(L_{i}, G\right)>0$, the selected path can benefit the primary pair with a higher effective bit rate, or a smaller delay, or both; when $U_{i}^{P}\left(L_{i}, G\right)<0$, the selected path degrades the performance of the primary pair. Thus, $U_{i}^{P}\left(L_{i}, G\right)$ can be viewed as the performance growing rate of the primary pair $\left\{\mathrm{PT}_{i}, \mathrm{PR}_{i}\right\}$.

When considering the cooperation from the view point of the SUs, the achievable throughput and the energy consumption are two key concerns. In other words, a SU aims at obtaining a high throughput for itself while consuming little energy for relaying the primary traffic. In cooperative transmissions, $\mathrm{SU}_{j}$ that relays for $\mathrm{PT}_{i}$ can get the following throughput profit:

$$
B_{j}(i, G)=T_{j}^{a}(i, G) R_{\left(j, j_{c}\right)}(i, G),
$$

where $T_{j}^{a}(i, G)$ is the obtained channel access time, and $R_{\left(j, j_{c}\right)}(i, G)=\left(C-\theta_{i} C\right) W \log _{2}\left(1+\operatorname{SNR}_{\left(j, j_{c}\right)}\right)$ is the effective bit rate from $\mathrm{SU}_{j}$ to its receiver $\mathrm{SU}_{j_{c}}$.

Meanwhile, $\mathrm{SU}_{j}$ costs a certain amount of energy for relaying, i.e., the energy cost, which is estimated by,

$$
Q_{j}(i, G)=T_{j}^{r}(i, G) P_{j},
$$

where $T_{j}^{r}(i, G)$ is the transmission time for relaying the PU traffic and $P_{j}$ is $\mathrm{SU}_{j}$ 's transmit power.

Accordingly, the payoff of $\mathrm{SU}_{j}$ working as the $i$-th primary pair's relay can be defined by:

$$
U_{j}^{S}(i, G)=\frac{B_{j}(i, G)}{\left[T_{j}^{a}(i, G)+T_{j}^{r}(i, G)\right] P_{j}} .
$$

From (9), $U_{j}^{S}(i, G)$ can be viewed as the transmission efficiency of a relay in terms of the number of bits per unit energy. Note that each $\mathrm{SU}_{j}$ is allowed to serve at most one primary pair and $U_{j}^{S}(i, G)=0$ if $\mathrm{SU}_{j}$ is not selected as a relay. We say a SU is "free" if it is not a relay for any PU.

\section{Cooperation Rules}

In order to set up the cooperation between the primary and secondary players, some rules are needed to regulate their behaviors, which are presented in the following:

- Traffic Conservation

$$
T_{j^{\prime}}^{r}(i, G) R_{\left(v_{k-1}, v_{k}\right)}=T_{j}^{r}(i, G) R_{\left(v_{k}, v_{k+1}\right)},
$$

in which $\mathrm{SU}_{j^{\prime}}=v_{k-1}$ and $\mathrm{SU}_{j}=v_{k}$. That is, the traffic received by a relay must be sent to the next hop.

- Proportional Fairness

A primary pair may cooperate with multiple relays; thus the assignment of the channel access time should meet a certain fairness criterion. In this game, the channel access time obtained by each relay is proportional to its energy consumption, i.e.,

$$
T_{j}^{a}(i, G)=\frac{Q_{j}(i, G)}{\sum_{k \in V_{L_{i}}} Q_{k}(i, G)} .
$$

\section{Player Strategies}

In the network formation game, the action of each player is reflected by the operation on links. Accordingly, the action space contains four different operations: (i) add a link; (ii) delete a link; (iii) replace a link via (i) and (ii); and (iv) null.

Note that in our cooperative CRN the primary players have higher priorities than the secondary players and they need to initiate the cooperation; on the other hand, secondary players are restricted to determine whether to accept the cooperation request from primary players or not. In other words, the primary players can perform their strategies in the game, and the secondary players are only allowed to passively react to the cooperation request without performing any strategy, i.e., the primary and the secondary players are the leaders and the followers, respectively. Particularly, the relay selection is carried out by PTs, forming paths from PTs to PRs. In this regard, it is not necessary for PRs to play strategies.

Definition 2: (Strategy Space) The strategy space of $\mathrm{PT}_{i}$ is defined as a set of triples $\mathcal{S}_{i}=\left\{s_{i}(j)=\right.$ $\left\langle+\left(v_{k}, \mathrm{SU}_{j}\right),+\left(\mathrm{SU}_{j}, v_{k+1}\right),-\left(v_{k}, v_{k+1}\right)\right\rangle\left|\mathrm{SU}_{j} \in(\mathcal{N} \cap V)\right\rangle$ $\left.V_{L_{i}}, v_{k}, v_{k+1} \in V_{L_{i}}\right\}$.

That is, the strategy of $\mathrm{PT}_{i}$ is a sequence of actions: add $\mathrm{SU}_{j}$ into its current path by forming two new links $\left(v_{k}, \mathrm{SU}_{j}\right)$ and $\left(\mathrm{SU}_{j}, v_{k+1}\right)$ and deleting the old link $\left(v_{k}, v_{k+1}\right)$. Let $G_{i,-i}$ be the network graph in which only $\mathrm{PT}_{i}$ is allowed to play a strategy. Accordingly, denote by $L_{i}+s_{i}(j)$ and $G_{i,-i}+s_{i}(j)$ the modified path and network graph after $\mathrm{PT}_{i}$ plays strategy $s_{i}(j)$.

Definition 3: (Beneficial Strategy) A strategy $s_{i}(j) \in \mathcal{S}_{i}$ is a beneficial strategy for $\mathrm{PT}_{i}$ if and only if $U_{i}^{P}\left(L_{i}+\right.$ $\left.s_{i}(j), G_{i,-i}+s_{i}(j)\right)>U_{i}^{P}\left(L_{i}, G_{i,-i}\right)$.

Definition 4: (Strategy Preference List) For $\mathrm{PT}_{i}$, a strategy preference list $\overline{\mathcal{S}}_{i}$ is the set of all its beneficial strategies 
sorted in a non-increasing order according to the corresponding payoff values.

Definition 5: (Best Strategy) A strategy $s_{i}\left(j^{*}\right) \in \mathcal{S}_{i}$ is the best strategy of $\mathrm{PT}_{i}$ if and only if $s_{i}\left(j^{*}\right)$ is in the first-place of the strategy preference list $\overline{\mathcal{S}}_{i}$.

Playing a beneficial strategy can enhance the payoff of $\mathrm{PT}_{i}$, motivating it to set up cooperative transmissions; while selecting the best strategy can maximize its payoff. We say $\mathrm{PT}_{i}$ has "incentive" if its strategy preference list is nonempty. In contrast, $\mathrm{PT}_{i}$ has only null action if its strategy preference list is empty.

\section{Distributed Dynamic Network Formation}

The objective of each player is to maximize its payoff via strategy selection. In this section, we propose a distributed algorithm to model the interaction among the players and to form a network structure for the cooperative CRN.

\section{A. Preference-Based Dynamic Formation Algorithm}

At the beginning of the game, the initial network graph $G_{0}$ contains no link $(E=\emptyset)$. During the distributed network formation process, each player maximizes its payoff via a myopic strategy selection scheme based on its strategy preference list without considering the future evolution of the network. The players' myopic behaviors are mainly resulted from the fact that it is difficult or costly to collect the information of the whole network to predict a long-term payoff. Therefore, players prefer to improve their short-term payoffs.

By taking into account the myopic player behavior and the dynamics of the network formation, we propose a distributed formation algorithm termed "PReference-bAsed Dynamic formAtion" (PRADA), which consists of a sequence of rounds with each containing multiple iterations. In order to determine a path towards $\mathrm{PR}_{i}, \mathrm{PT}_{i}$ exploits an entity called announcer to find out the next-hop relay. Each $\mathrm{PT}_{i}$ selects the next-hop relay according to the following steps:

\section{1) Payoff Negotiation}

The announcer of $\mathrm{PT}_{i}$ announces its cooperation information, including the positions of the $\mathrm{PT}_{i}$ and $\mathrm{PR}_{i}$, the number of sub-carriers in the leased sub-channel, the bandwidth of a sub-carrier, and the number of relays on the current path, to its SU neighbors. Then, each neighboring $\mathrm{SU}$ calculates the payoff obtained from $\mathrm{PT}_{i}$ and feedbacks the cooperation information including its relay time, position, and transmission power to $\mathrm{PT}_{i}$.

\section{2) Offer Provision}

After receiving the replies from all neighboring SUs, $\mathrm{PT}_{i}$ computes the payoff value for each strategy in $\mathcal{S}_{i}$ and makes the corresponding strategy preference list $\overline{\mathcal{S}}_{i}$. If $\overline{\mathcal{S}}_{i}=\emptyset, \mathrm{PT}_{i}$ does not have any incentive to cooperate and it ends its turn; otherwise, it sends an offer to $\mathrm{SU}_{j^{*}}$, which corresponds to the best strategy $s_{i}\left(j^{*}\right)$, with a decision probability $\tau=1-\varepsilon(0<\varepsilon<1)$, and then removes $s_{i}\left(j^{*}\right)$ from $\overline{\mathcal{S}}_{i}$. With this removal, the best strategy of $\mathrm{PT}_{i}$ changes after each offer provision and a $\mathrm{SU}$ receives the offer from $\mathrm{PT}_{i}$ at most once during each round.

3) Cooperation Agreement

We say that $\mathrm{PT}_{i}$ gets into cooperation with $\mathrm{SU}_{j^{*}}$ if its offer is accepted and it is out of cooperation if it is rejected by $\mathrm{SU}_{j^{*}}$.

If $\mathrm{SU}_{j^{*}}$ is free currently, it accepts the cooperation offer with the decision probability $\tau=1-\varepsilon$; otherwise, $\mathrm{SU}_{j}$ * compares the payoff $U_{j^{*}}^{S}\left(i, G_{i,-i}+s_{i}\left(j^{*}\right)\right)$ obtained from $\mathrm{PT}_{i}$ with its current best payoff $U_{j^{*}}^{S}\left(i^{\prime}, G_{i,-i}\right)$ offered by $\mathrm{PT}_{i^{\prime}}$. If $U_{j^{*}}^{S}\left(i, G_{i,-i}+s_{i}\left(j^{*}\right)\right)>U_{j^{*}}^{S}\left(i^{\prime}, G_{i,-i}\right)$, $\mathrm{SU}_{j^{*}}$ accepts $\mathrm{PT}_{i}$ 's offer with the probability $\tau=1-\varepsilon$ and $\mathrm{PT}_{i^{\prime}}$ becomes out of cooperation; otherwise, $\mathrm{SU}_{j}$ * accepts $\mathrm{PT}_{i}$ 's offer with the probability $\varepsilon$; and if rejected, $\mathrm{PT}_{i}$ is out of cooperation and thus goes to the step 2.

Instead of a deterministic decision procedure, players make a decision with some randomness in the network formation process in which links may be added or deleted via some exogenous stimulus or simply by error of the player with a small "mutation" probability $\varepsilon$.

The turn of $\mathrm{PT}_{i}$ continues until either $\mathrm{PT}_{i}$ gets into cooperation with some $\mathrm{SU}$ or $\mathrm{PT}_{i}$ 's strategy preference list becomes empty. After $\mathrm{PT}_{i}$ ends its turn, the PT that is still out of cooperation but has a nonempty strategy preference list is selected. The iteration of a round terminates whenever any one of the following two conditions holds: (i) Every PT with incentives has got into cooperation with some relay in its turn, which implies that each PT can increase its payoff with a beneficial strategy or even the best strategy. (ii) The strategy preference lists of the PTs that are out of cooperation are empty, which indicates that these PTs cannot find out a beneficial strategy or all their offers are rejected by the SUs.

The announcer of $\mathrm{PT}_{i}$ places a critical role in identifying a right relay. Initially, $\mathrm{PT}_{i}$ is the announcer and the path contains only the primary pair $\left\{\left(\mathrm{PT}_{i}, \mathrm{PR}_{i}\right)\right\}$. During the execution of our algorithm, the announcer is adjusted as follows: if a new relay is added into the path, the new relay should act as the announcer; if a relay exits the path, the previous-hop player of the relay should act as the announcer. If $\mathrm{PR}_{i}$ is arranged to be the announcer, $\mathrm{PR}_{i}$ turns over the announcement work to $\mathrm{PT}_{i}$, and $\mathrm{PT}_{i}$ suspends its strategy selection until a relay leaves its path; if $\mathrm{PT}_{i}$ 's offer is not accepted by any $\mathrm{SU}$ at the end of a round, the next-hop player of the current announcer in the path should work as the announcer for $\mathrm{PT}_{i}$.

Let $G_{x}$ be the network graph output at round $x \quad(x=$ $1,2, \cdots)$. As the round goes on, there is a sequence of network graphs, i.e., $\left\{G_{0}, G_{1}, G_{2}, \cdots, G_{x}, \cdots\right\}$.

The PRADA algorithm is summarized in Alg. 1, in which several variables are employed to record the states of the players and the network graphs: $\mathcal{M}_{T}$ is a set of all PTs, $\overline{\mathcal{M}}_{T}$ is a subset of $\mathcal{M}_{T}$ to track the cooperation states of the PTs, and $\rho$ is the threshold of the number of rounds.

Theorem 1: For each round of the PRADA algorithm (lines 5-30 in Alg. 1), the number of iterations is at most $M N$ when $\varepsilon=0$.

Proof: When $\varepsilon=0$, the strategy selection is deterministic. In the PRADA algorithm, each iteration consists of a PT providing the cooperation offer to a SU to which the PT has never sent the offer before. Since a PT sends an offer to each SU at most once and there are only $N$ SUs in the network, a PT can send the offer at most $N$ times. For $M$ PTs, it takes at most $M N$ times to complete the offer provision process. Therefore, a round can terminate after at most $M N$ iterations. 


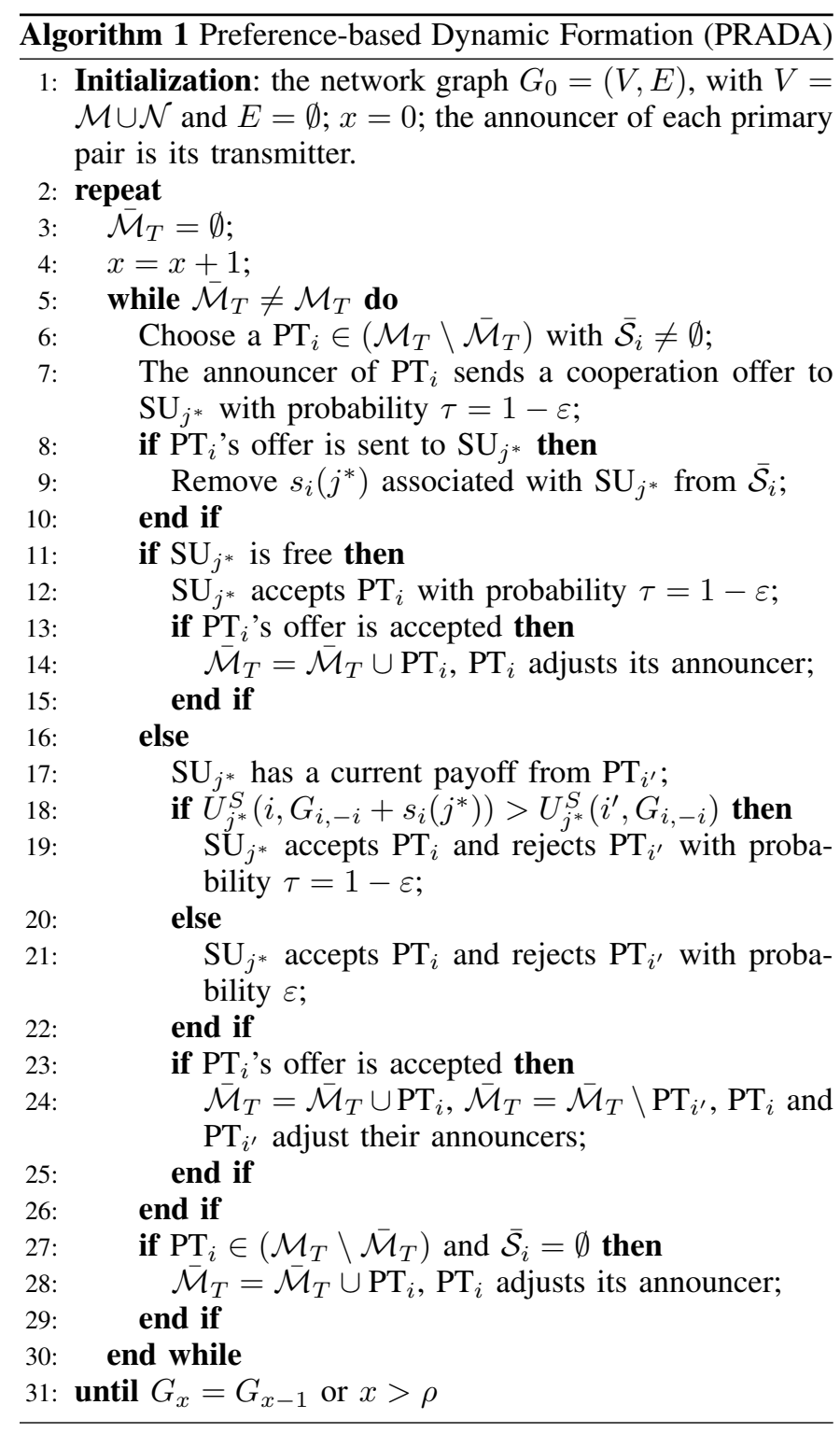

Based on the PRADA algorithm, it can be shown that our network formation game has the following properties.

Property 1: A network graph $G$ of the network formation game proposed in Section IV is a directed graph consisting of at most $M$ player-disjoint paths.

Property 1 is the direct result of two assumptions in our model: the first one states that each primary transmitter $\mathrm{PT}_{i}$ has its distinct receiver $\mathrm{PR}_{i}$ and the second one indicates that a relay is allowed to serve at most one of the $M$ paths.

Property 2: Given a network graph $G$ of the network formation game, the total bandwidth utility of all relays can achieve its maximum value if all relays' receivers are fixed.

For property 2, since the effective bit rate of each relay is a constant in a given network graph $G$ and the channel access time is allocated according to proportional fairness, the total bandwidth utility of all relays can reach its maximum according to the derivation in [17], [18].

Property 3: The process of the PRADA algorithm is an irreducible and aperiodic Markov chain.

In the PRADA algorithm, each player selects its myopic best strategy with a decision probability $\tau=1-\varepsilon$, where $\varepsilon$ is a mutation probability. According to [10], [11], [19], for any $0<\varepsilon<1$, the process of PRADA defines an irreducible and aperiodic Markov chain, and thus has a unique invariant stationary distribution. That is, the network $G_{x}$, the output of round $x$, is a sate of a Markov chain and $x=1,2, \cdots$ (i.e., $\rho \rightarrow \infty)$ is a discrete time period. As $\varepsilon \rightarrow 0$, the stationary distribution converges to a unique limiting stationary distribution. Moreover, by utilizing the PRADA algorithm, the network formation process can converge to a stable network graph, which is analyzed in the next subsection.

\section{B. Path Stable Equilibrium}

The PRADA algorithm allows all players to perform a local strategy selection based on their strategy preference lists. In order to examine the stability of the network graph generated by PRADA, we first present two stable equilibrium concepts: global-path stability and local-path stability.

Definition 6: (Global-Path Stable) A directed network graph $G$ is global-path stable if for every primary pair $\left\{\mathrm{PT}_{i}, \mathrm{PR}_{i}\right\} \in \mathcal{M}, U_{i}^{P}\left(L_{i}, G_{i,-i}\right) \geq U_{i}^{P}\left(L_{i}+s_{i}(j), G_{i,-i}+\right.$ $\left.s_{i}(j)\right)$, for $\forall s_{i}(j) \in \overline{\mathcal{S}}_{i}$.

Definition 7: (Local-Path Stable) A directed network graph $G$ is local-path stable if for any primary pair $\left\{\mathrm{PT}_{i}, \mathrm{PR}_{i}\right\} \in \mathcal{M}$, the following condition holds:

$$
\begin{aligned}
& U_{i}^{P}\left(L_{i}, G\right)<U_{i}^{P}\left(L_{i}^{\prime}, G^{\prime}\right) \\
\Rightarrow \quad \exists j \in \triangle V, U_{j}^{s}(i, G)>U_{j}^{s}\left(i^{\prime}, G^{\prime}\right) & \text { or } U_{j}^{s}\left(i^{\prime}, G\right)>U_{j}^{s}\left(i, G^{\prime}\right),
\end{aligned}
$$

where $\mathrm{PT}_{i^{\prime}} \in \mathcal{M}_{T}\left(i^{\prime} \neq i\right), \triangle V=\left(V_{L_{i}} \cup V_{L_{i}^{\prime}}\right) \backslash\left(V_{L_{i}} \cap V_{L_{i}^{\prime}}\right)$, $L_{i} \neq L_{i}^{\prime}$, and $G \neq G^{\prime}$.

In a global-path stable network, no PT has an incentive to play the strategies that can help to obtain a better payoff. However, in a local-path stable network, a PT is allowed to change its path for a higher payoff only if there is no decrease in the payoffs of the relays that are newly added or deleted. In other words, the global-path stability takes into account all PTs and all relays from the view point of the whole network, while the local-path stability only focuses on a path and some relays within a certain local area.

Theorem 2: The network graph $G_{x}$ output by the $x$-th round of the algorithm PRADA is local-path stable.

Proof: We consider the following two cases.

(1) Case 1: $\varepsilon=0$. If there is instability with respect to the network graph $G_{x}$, at least two paths are involved. Without loss of generality, let $\mathrm{PT}_{i}$ and $\mathrm{PT}_{j}$ be the transmitters of the $i$-th path and the $j$-th path, respectively. $\mathrm{SU}_{j}$ is a relay of the $j$ th path with the following assumptions: (i) $\mathrm{PT}_{i}$ can get a better payoff by adding $\mathrm{SU}_{j}$; and (ii) $\mathrm{SU}_{j}$ can get a better payoff by accepting the offer of $\mathrm{PT}_{i}$. Before the end of the round, if $\mathrm{PT}_{i}$ does not send an offer to $\mathrm{SU}_{j}$, the payoff of cooperating with $\mathrm{SU}_{j}$ is lower than $\mathrm{PT}_{i}$ 's current strategy, which contradicts to the assumption (i). Within the round, if $\mathrm{PT}_{i}$ has sent an offer to $\mathrm{SU}_{j}$ but was rejected by $\mathrm{SU}_{j}$ because $\mathrm{SU}_{j}$ is in favor of another PT that can provide a higher payoff. As the iteration goes, $\mathrm{SU}_{j}$ will obtain the highest payoff at the end of the round. That is, the payoff provided by $\mathrm{PT}_{j}$ is higher than that 


$$
U_{i}^{P}\left(L_{i}, G\right)= \begin{cases}\frac{R_{\left(v_{k}, v_{k+1}\right)}(G)}{R_{i}(G)}-\frac{\sum_{\left(v_{k}, v_{k+1}\right) \in L_{i}} D_{\left(v_{k}, v_{k+1}\right)}(G)}{D_{i}(G)}, & \text { if } R_{\left(v_{k}, v_{k+1}\right)}(G)=R_{L_{i}}(G) \\ \frac{R_{L_{i}}(G)}{R_{i}(G)}-\frac{\sum_{\left(v_{k}, v_{k+1}\right) \in L_{i}} D_{\left(v_{k}, v_{k+1}\right)}(G)}{D_{i}(G)}, & \text { otherwise. }\end{cases}
$$

provided by $\mathrm{PT}_{i}$. This result contradicts to the assumption (ii). Therefore, $G_{x}$ is local-path stable.

(2) Case 2: $\varepsilon>0$. As analyzed by Theorem 1, the finite number of iterations at each round is $M N$, i.e., all possible networks output at the end of the iterations during each round constitute a sequence of at most $M N$ networks. Since each player can obtain a strictly better payoff when performing a strategy with a probability $\tau=1-\varepsilon$, the probability that the dynamic process does not reach a local-path stable network after $M N$ iterations is not more than $\varepsilon$. Then, $G_{x}$ is not a local-path stable network after $\alpha(M N)$ iterations with a probability at most $\varepsilon^{\alpha}$, where $\alpha$ is a positive integer. As $\alpha$ becomes larger, $\varepsilon^{\alpha} \rightarrow 0$. Thus, the process converges to a local-path stable network $G_{x}$ with probability 1 .

Therefore we can conclude that $G_{x}$ is a local-path stable network.

Property 4: In the proposed network formation game, the global-path stability is equivalent to the Nash equilibrium.

When a network graph $G$ is global-path stable, no PT can improve its payoff by unilaterally changing its path based on the strategy preference list, which is a Nash equilibrium in the network formation game.

Theorem 3: The PRADA algorithm can converge to a global-path stable network as $\varepsilon \rightarrow 0$ and $\rho \rightarrow \infty$.

Proof: According to Properties 3 and 4 , as $\varepsilon \rightarrow 0$ and $\rho \rightarrow \infty$, the convergence toward a global-path stable network for the PRADA algorithm is equivalent to the existence of at least one global-path stable network $G$ in the proposed network formation game (i.e., a Nash equilibrium exists in the relay selection game among the PTs).

Given $M$ primary pairs and $N$ SUs, the beneficial strategy space for all PTs $\overline{\mathcal{S}}=\bigcup_{i=1}^{M} \overline{\mathcal{S}}_{i}, 0<|\overline{\mathcal{S}}| \leq M N$ !, is a nonempty and compact space (if $|\overline{\mathcal{S}}|=0, G$ is always a globalpath stable network graph). To prove the existence of at least one network $G$ with Nash equilibrium, it is sufficient to prove that the payoff function of PUs, $U_{i}^{P}(\cdot)$, is continuous and concave in the compact space $\overline{\mathcal{S}}$ [20], [21]. For the effective bit rate $R_{\left(v_{k}, v_{k+1}\right)}(G)$ of each link $\left(v_{k}, v_{k+1}\right)$ added by a strategy in $\overline{\mathcal{S}}$, the payoff function (6) can be rewritten by (12).

From $(12), U_{i}^{P}(\cdot)$ is obviously continuous in $\overline{\mathcal{S}}$. Then, we take the second order derivative with respect to $R_{\left(v_{k}, v_{k+1}\right)}(G)$ to prove its concavity.

$\frac{\partial^{2} U_{i}^{P}\left(L_{i}, G\right)}{\partial^{2} R_{\left(v_{k}, v_{k+1}\right)}(G)}=-\frac{\operatorname{Len}\left[R_{\left(v_{k}, v_{k+1}\right)}^{3}(G)+\left(R_{\left(v_{k}, v_{k+1}\right)}(G)-\lambda_{i} L e n\right)^{3}\right]}{D_{i}(G) R_{\left(v_{k}, v_{k+1}\right)}^{3}(G)\left[R_{\left(v_{k}, v_{k+1}\right)}(G)-\lambda_{i} L e n\right]^{3}}$.

Since $\mu_{\left(v_{k}, v_{k+1}\right)}=\frac{R_{\left(v_{k}, v_{k+1}\right)}(G)}{L e n}>\lambda_{i}$, we have $\frac{\partial^{2} U_{i}^{P}\left(L_{i}, G\right)}{\partial^{2} R_{\left(v_{k}, v_{k+1}\right)}(G)}<0$, i.e., $U_{i}^{P}(\cdot)$ is concave in $\overline{\mathcal{S}}$. Therefore, a Nash equilibrium exists in the proposed network formation game. It follows that the PRADA algorithm can converge to a global-path stable network as $\varepsilon \rightarrow 0$ and $\rho \rightarrow \infty$.

The theoretical analysis presented above shows that the network formation game proposed in the PRADA algorithm can achieve the global-path stability. However, to consider a tradeoff between the stability and the time complexity in realworld applications, we let $\rho$ be a finite integer to set up a termination condition for the PRADA algorithm (line 31 in Alg. 1): if $G_{x}=G_{x-1}, G_{x}$ is global-path stable and is the final output; otherwise, if the number of rounds $x$ exceeds $\rho$, the process of the dynamic network formation stops and outputs the network graph $G_{x}$ as the final result.

\section{NUMERICAL EXPERIMENT}

\section{A. Methodology}

In our numerical experiment, we simulate an IEEE 802.16 OFDMA network located in an area of $5000 \times 5000 \mathrm{~m}^{2}$. The primary spectrum contains 256 sub-carriers centered at the 5 $\mathrm{GHz}$ band and 200 of them are used for data traffic with each having a bandwidth of $312.5 \mathrm{KHz}$ [22], [23]. We uniformly and randomly deploy 10 primary pairs within the network. Each primary transmitter is assigned a sub-channel containing 20 sub-carriers.

To check the impact of the number of relays, we vary the number of SUs from 10 to 50 . We also set the transmit power of the users to different levels ranging from $10 \mathrm{~mW}$ to $100 \mathrm{~mW}$, to examine the impact of the transmission range. The mutation probability $\varepsilon$ is selected from $\{0.1,0.01,0.001,0.0001,0\}$ to analyze its impact on the performance of the PRADA algorithm. Note that the mutation probability should be sufficiently small because users typically target a better payoff. The values of other parameters are listed in TABLE I.

TABLE I

NumeriCAL EXPERIMENT PARAMETERS

\begin{tabular}{|l|l|}
\hline \multicolumn{1}{|c|}{ Parameter } & \multicolumn{1}{c|}{ Value } \\
\hline Noise & $10^{-10} \mathrm{~mW}$ \\
\hline Shadowing Factor & 4 \\
\hline Bandwidth Requirement $\left(\theta_{i}\right)$ & {$[0.5,1]$} \\
\hline Packet Size $($ Len $)$ & 1024 bits \\
\hline Packet Arrival Rate $\left(\lambda_{i}\right)$ & $(0,1)$ \\
\hline Threshold of the Number of Rounds $(\rho)$ & 20 \\
\hline
\end{tabular}

We investigate the performance of the PRADA algorithm based on the following performance metrics:

- The per-user payoff, profit, and cost.

- The average number of relays on each path.

- The relay utilization, which is defined to be the ratio of the total number of relays to the total number of SUs, indicating the percentage of the SUs involved in cooperative transmissions.

- The number of rounds and the corresponding statistics such as the maximum and minimum number of rounds in PRADA, reflecting the stability of the resulted network graphs and the convergence speed of the PRADA algorithm. 
- The total number of iterations in all rounds, which reflects the time complexity of the PRADA algorithm.

\section{B. Player Performance}

We first report the numerical results from the view point of the users' performance with $\varepsilon=0.1$. For statistical confidence, the averaged results of 100 runs are reported.

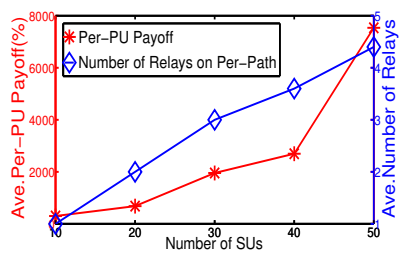

(a) Impact of Number of SUs

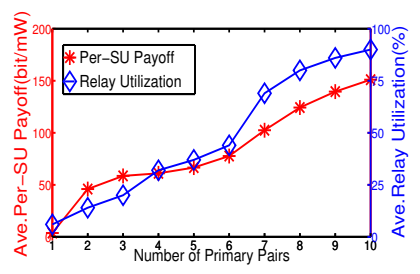

(b) Impact of Number of Primary Pairs
Fig. 2. Impact of User Density.

Fig. 2(a) reports the average per-PU payoff and average number of relays each primary pair selects when the number of SUs varies. In this study, the transmit powers of the 10 primary transmitters and the SUs are fixed to $100 \mathrm{~mW}$. We observe that as more and more SUs join the network, more potential relays can be employed by the PUs to improve performance. In particular, the blue curve shows that the average number of relays taken by a primary transmitter becomes larger when the number of SUs increases, indicating that more SUs get into cooperation with the PUs. Similarly, as shown in Fig. 2(b) which reports the average per-PU payoff and average relay utilization when in total 50 SUs exist. We notice that more SUs can obtain opportunities for cooperative transmissions when the number of PUs goes up, increasing the corresponding relay utilization (see the blue curve).

Fig. 3 presents the impact of the transmit power on cooperative transmissions for a scenario that contains 10 primary pairs and 50 SUs. As defined in (6), the PU's profit is the bit rate growth rate, its cost is the corresponding delay growth rate, and its payoff is equal to the profit minus the cost. For a SU (or a relay), the profit is the achievable throughput, the cost is the energy consumption for relaying, and the corresponding payoff is defined to be the ratio of the profit to the cost (see (9)). The impact of the transmit power can be demonstrated from the following two aspects.

On one hand, a larger transmit power can enlarge the transmission range, providing more cooperation opportunities for the PUs and the SUs. From Fig. 3(a), it can be observed that the average number of relays is only 2.4 when the power is set to $10 \mathrm{~mW}$ while this value increases to 4.5 when the transmit power is $60 \mathrm{~mW}$. Thus by utilizing more SUs as relays, both the PUs and the SUs can obtain better payoff.

On the other hand, if no more relay can be invited to participate in the cooperative transmissions, increasing the transmit power can reduce the users' payoff. For example, in Fig. 3, the average number of relays on a path remains 4.5 when the power ranges from 60 to $120 \mathrm{~mW}$. By enhancing the transmit power, a primary pair can directly communicate with a higher bit rate and a shorter delay, decreasing the payoff from the relay service if no new relay is added. In particular, without adding any new relay, Fig. 3(b) shows that the PU profit gradually declines while the corresponding cost gradually increases as the transmit power becomes larger, leading to a reduction in the PU's payoff. In addition, from the view point of the SU, when the transmit power goes up, the growth rate of the cost is higher than that of the profit due to the corresponding mathematical properties (see (7) and (8)), resulting in a decrease in the payoff as shown in Fig. 3(c).

\section{Algorithm Performance}

In this subsection, we analyze the performance of the PRADA algorithm by examining the impact from the number of SUs and the value of the mutation probability $\varepsilon$. The results are the average of 100 runs. There are in total 10 primary pairs and the transmit power is fixed to $100 \mathrm{~mW}$.

The impact of the number of SUs on the PRADA algorithm is reported in Fig. 4(a), where $\varepsilon=0.1$. We particularly report the maximum and the minimum number of rounds, which indicate the worst case and the best case of the PRADA algorithm, respectively. Although the number of rounds (including the maximum number, the average number, and the minimum number) gradually goes up as the number of SUs becomes larger, it is smaller than the threshold $\rho=20$ in our study. For example, when there are $50 \mathrm{SUs}$, the maximum number of rounds is 18 , which is smaller than $\rho=20$. This confirms that the global-path stability does exist in our proposed network formation game, as analyzed in Theorem 3.

Fig. 4(b) presents the convergence speed of the PRADA algorithm when there are 50 SUs in the network and $\varepsilon$ varies. As illustrated by Property 3 , the stochastically dynamic formation process can converge to a finite sate when $\varepsilon \rightarrow 0$. Moreover, a smaller $\varepsilon$ can accelerate the convergence, since the lower mutation probability can reduce the randomness degree in the strategy selection and generate a stable network with a higher probability. From Fig. 4(b), it can be seen that the PRADA algorithm converges to a global-path stable network graph via fewer and fewer rounds as $\varepsilon$ decreases from 0.1 to 0 . In particular, the PRADA algorithm needs only 9 rounds to converge when $\varepsilon=0.0001$ and $\varepsilon=0$.

Fig. 4(c) demonstrates the time complexity of the algorithm in terms of the total number of iterations of all rounds. As $\varepsilon$ goes down, the number of total iterations is reduced due to a higher convergence speed, which is consistent with the analysis in Fig. 4(b). On the other hand, when $\varepsilon=0$, the maximum number of iterations within a round for a network scenario of 50 secondary users is about 64, which is in accordance with the conclusion of Theorem 1 that a round has at most $M N$ iterations when $\varepsilon=0$.

\section{CONCLUSION}

In cooperative cognitive radio networks, the cooperation between the PU and the SU plays an important role in the performance improvement. In this paper, we apply a novel network formation game to form a multi-hop path between the primary transmitter and its receiver by employing the SUs as relays. We first construct a cooperation framework FTCO, in which a relay can simultaneously forward the primary traffic in the cooperative sub-channel and send the secondary traffic in the leased sub-channel without any interference. Then, we utilize the network formation game to model the multi-hop 


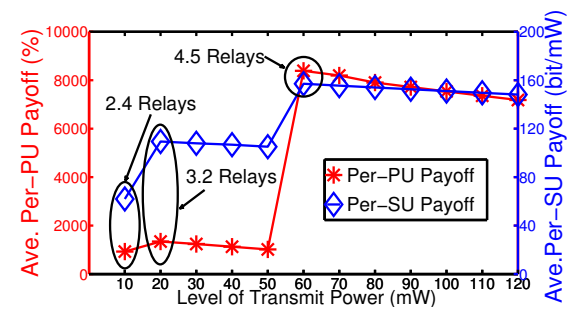

(a) User's Payoff vs. Transmit Power

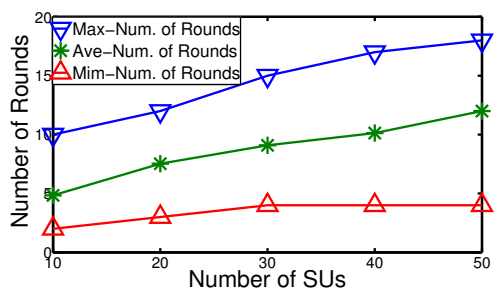

(a) Impact of Number of SUs

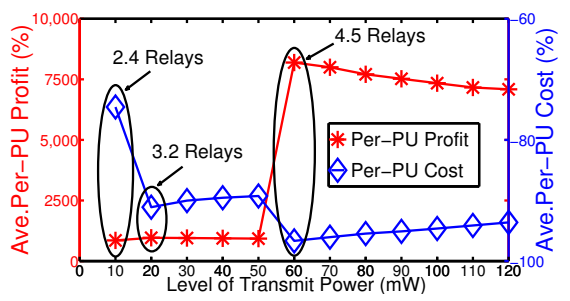

(b) PU's Profit and Cost vs. Transmit Power

Fig. 3. Impact of Transmit Power

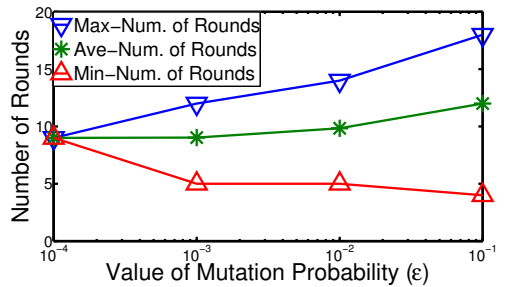

(b) Impact of Mutation Probability

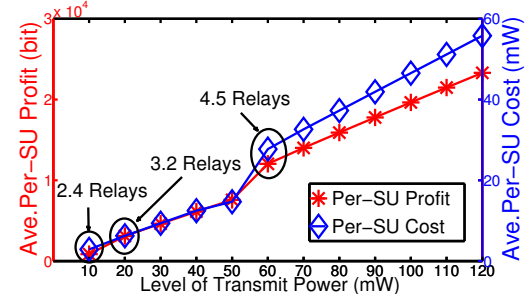

(c) SU's Profit and Cost vs. Transmit Power

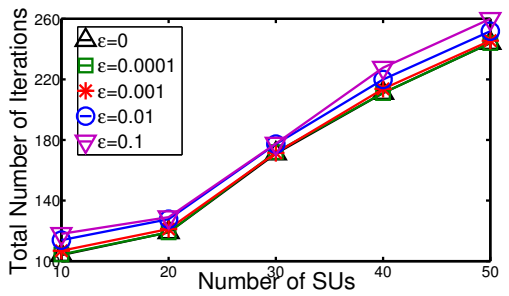

(c) Time Complexity of PRADA

Fig. 4. Stability and Convergence.

relay selection by taking into account the effective bit rate and the delay of the primary transmissions, and the achievable throughput and energy consumption of the relays. To find out a stable network, we design a distributed stochastically dynamic algorithm PRADA, which is theoretically proved that the output graph of PRADA is global-path stable. Numerical results demonstrate that both the PU and the SU can obtain significant payoffs in multi-hop relaying cooperation and that the proposed algorithm PRADA can effectively converge to a global-path stable network graph.

\section{ACKNOWLEDGMENT}

The authors would like to thank the support from the National Science Foundation of the US (CNS-1162057), the National Natural Science Foundation of China (Grant No. 61272503 and 61272505), and the Fundamental Research Funds for the Central Universities of China (W12JB00390).

\section{REFERENCES}

[1] T. Jing, S. Zhu, H. Li, X. Cheng, and Y. Huo, "Cooperative relay selection in cognitive radio networks," in IEEE INFOCOM Mini-Conference, 2013.

[2] O. Simeone, I. Stanojev, S. Savazzi, Y. Bar-Ness, U. Spagnolini, and R. Pickholtz, "Spectrum leasing to cooperating secondary ad hoc networks," IEEE Journal on Selected Areas in Communications, vol. 26, no. 1, pp. 203-213, January 2008.

[3] J. Zhang and Q. Zhang, "Stackelberg game for utility-based cooperative cognitive radio networks," in ACM MobiHoc, May 2009, pp. 23-31.

[4] D. Yang, X. Fang, and G. Xue, "Truthful auction for cooperative communications," in ACM MobiHoc, May 2011, pp. 89-98.

[5] D. Li, Y. Xu, X. Wang, and M. Guizani, "Coalitional game theoretic approach for secondary spectrum access in cooperative cognitive radio networks," IEEE Transactions on Wireless Communications, vol. 10, no. 3, pp. 844-856, March 2011.

[6] H. Xu and B. Li, "Efficient resource allocation with flexible channel cooperation in ofdma cognitive radio networks," in IEEE INFOCOM, March 2010, pp. 561-569.

[7] H. Li, X. Cheng, K. Li, X. Xing, and T. Jing, "Utility-based cooperative spectrum sensing scheduling in cognitive radio networks," in IEEE INFOCOM Mini-Conference, 2013.
[8] A. Lodhi, A. Dhamdhere, and C. Dovrolis, "Genesis: An agent-based model of interdomain network formation, traffic flow and economics," in IEEE INFOCOM, March 2012, pp. 1197-1205.

[9] H. Nama, N. Mandayam, and R. Yates, "Network formation among selfish energy-constrained wireless devices," in IEEE INFOCOM, April 2008, pp. 753-761.

[10] M. O. Jackson and A. Watts, "The evolution of social and economic networks," Journal of Economic Theory, vol. 106, no. 2, pp. 265-295 October 2002.

[11] — "On the formation of interaction networks in social coordination games," Games and Economic Behavior, vol. 41, no. 2, pp. 265-291, November 2002.

[12] R. Johari, S. Mannor, and J. N. Tsitsiklis, "A contract-based model for directed network formation," Games and Economic Behavior, vol. 56, no. 2, pp. 201-224, August 2006.

[13] W. Saad, Z. Han, T. Başar, M. Debbah, and A. Hjørungnes, "Network formation games among relay stations in next generation wireless networks," IEEE Transactions on Communications, vol. 59, no. 9, pp. 2528-2542, September 2011.

[14] X. Chen, T. Jing, Y. Huo, W. Li, X. Cheng, and T. Chen, "Achievable transmission capacity of cognitive radio networks with cooperative relaying," in Crowncom, June 18-20 2012.

[15] W. Li, X. Cheng, T. Jing, Y. Cui, K. Xing, and W. Wang, "Spectrum assignment and sharing for delay minimization in multi-hop multi-flow crns," IEEE Journal on Selected Areas in Communications (JSAC), Special Issue on Cognitive Radio, to appear, 2013.

[16] D. Bertsekas and R. Gallager, Data Networks. Prentice-Hall, 1987.

[17] W. Li, S. Wang, Y. Cui, X. Cheng, R. Xin, M. A. Al-Rodhaan, and A. Al-Dhelaan, "AP association for proportional fairness in multi-rate WLANs," IEEE/ACM Transactions on Networking, submitted, 2012.

[18] W. Li, Y. Cui, S. Wang, and X. Cheng, "Approximate optimization for proportional fair AP association in multi-rate WLANs," in 5th Int. Cof. WASA, August 2010, pp. 36-46.

[19] S. Goyal and F. Vega-Redondo, "Network formation and social coordination," Games and Economic Behavior, vol. 50, no. 2, pp. 178-207, February 2005.

[20] J. John F. Nash, "Equilibrium points in N-person games," Proc. of the National Academy of Science of the USA, vol. 36, no. 1, pp. 48-49, 1950 .

[21] G. Debreu, "A social equilibrium existence theorem," Proc. of the National Academy of Science of the USA, vol. 38, no. 10, pp. 886-893, October 1952.

[22] "IEEE Std 802.16 air interface for broadband wireless access systems," IEEE Standard 802.16, 2009.

[23] "IEEE Std 802.16h air interface for broadband wireless access systems: Improved coexistence mechanisms for license-exempt operation," IEEE Standard 802.16h, 2010. 